\title{
Production of Human Cu,Zn SOD with Higher Activity and Lower Toxicity in E. coli via Mutation of Free Cysteine Residues
}

\author{
Kun Zhang, ${ }^{1}$ Yuejuan Zhang, ${ }^{1}$ Jing Zi, ${ }^{1}$ Xiaochang Xue, ${ }^{2}$ and Yi Wan \\ ${ }^{1}$ Microbiology Institute of Shaanxi, Xi'an 710043, China \\ ${ }^{2}$ State Key Laboratory of Cancer Biology, Department of Biopharmaceutics, School of Pharmacy, \\ Fourth Military Medical University, Xian 710032, China \\ Correspondence should be addressed to Xiaochang Xue; xue_xiaochang@yahoo.com and Yi Wan; wanyi6565@sina.com
} Received 9 October 2016; Revised 13 December 2016; Accepted 24 January 2017; Published 16 February 2017 Academic Editor: Viness Pillay

Copyright ( $\odot 2017$ Kun Zhang et al. This is an open access article distributed under the Creative Commons Attribution License, which permits unrestricted use, distribution, and reproduction in any medium, provided the original work is properly cited.

\begin{abstract}
Although, as an antioxidant enzyme, human $\mathrm{Cu}, \mathrm{Zn}$ superoxide dismutase 1 (hSOD1) can mitigate damage to cell components caused by free radicals generated by aerobic metabolism, large-scale manufacturing and clinical use of hSOD1 are still limited by the challenge of rapid and inexpensive production of high-quality eukaryotic hSOD1 in recombinant forms. We have demonstrated previously that it is a promising strategy to increase the expression levels of soluble hSOD1 so as to increase hSOD1 yields in $E$. coli. In this study, a wild-type hSOD1 (wtSOD1) and three mutant SOD1s (mhSOD1s), in which free cysteines were substituted with serine, were constructed and their expression in soluble form was measured. Results show that the substitution of Cys111 (mhSOD1/C111S) increased the expression of soluble hSOD1 in E. coli whereas substitution of the internal Cys6 (mhSOD1/C6S) decreased it. Besides, raised levels of soluble expression led to an increase in hSOD1 yields. In addition, mhSOD1/C111S expressed at a higher soluble level showed lower toxicity and stronger whitening and antiradiation activities than those of wtSOD1. Taken together, our data demonstrate that C111S mutation in hSOD1 is an effective strategy to develop new SOD1-associated reagents and that $\mathrm{mhSOD1/C111S}$ is a satisfactory candidate for large-scale production.
\end{abstract}

\section{Introduction}

Human $\mathrm{Cu}, \mathrm{Zn}$ superoxide dismutase 1 (hSOD1) is an antioxidant enzyme which protects cells against the oxidative by-products of aerobic metabolism through catalyzing the dismutation of superoxides into oxygen and hydrogen peroxide [1-3]. Given the potential importance of hSOD1 in medical applications such as limiting the detrimental effects of reactive oxygen species (ROS) in ischemia-reperfusion injury [4] and protecting cells from radiotherapy $[5,6]$, the development of efficient and viable production systems to obtain hSOD1 with higher yields or activity has received much attention for many years $[7,8]$.

Although the E. coli expression system is one of the most widely used systems for large-scale production of recombinant proteins, expressed proteins often form inclusion bodies without biological activity, which thus puts a constraint to limit their yields [9-11]. To remove this constraint, we have previously expressed hSOD1 in E. coli and purified it from both the inclusions and the cell lysate supernatant. The specific activity of the recombinant hSOD1 purified from the supernatant was higher than that from the inclusions by approximately 3 -fold (unpublished data). In view of this, we assume that increasing the hSOD1 soluble expression level in E. coli may probably improve its specific activity and yields.

Disulfide bond exchange and intermolecular disulfide bond formation can both produce insoluble protein aggregates and affect protein activity [12]. For example, the EC1 domains of E-cadherins with a single free Cys residue can form a covalent dimer, thus inducing the precipitation of the proteins [13]. hSOD1 is a dimeric metalloprotein composed of identical non-covalently linked subunits [14], each of which contains 4 cysteine residues. Cys57 and Cys148 form an intrasubunit disulfide bond whereas Cys6 and Cys111 remain unbonded. Cys6 is buried within the protein whereas Cys111 is on its surface near the dimer interface $[15,16]$. It has been shown that incorrect localization of cysteine in hSOD1 has caused protein oligomerization in ALS mice [17]. Cozzolino 
et al. studied the mechanism of the aberrant aggregation of mutant SOD1 (mutSOD1), which is closely associated with familial amyotrophic lateral sclerosis (FALS) [18]. Through mutating each of the four cysteine residues in SOD1 and analyzing the solubility and aggregation of those SOD1s in NSC-34 cells, they found that covalent disulfide cross-linking plays a pivotal role in the production of mutSOD1 aggregates and removal of Cys111 remarkably reduces the ability of FALS-related mutSOD1s to form aggregates and improves the viability of NSC-34 cells. However, considering that the recombinant protein expression efficiency and redox environment of microorganisms differ from those of mammalian cells, it is still unknown whether the cysteine mutation in hSOD1 can reduce aggregates formation and improve soluble expression in E. coli.

Given these observations, in the present study, we constructed three hSOD1 mutants, in which free cysteines were substituted with serine. Two single cysteine mutants were produced by replacing either Cys6 (mhSOD1/C6S) or Cys111 (mhSOD1/C111S) with serine, and one double cysteine mutant was produced by replacing both Cys6 and Cys111 with serine (mhSOD1/C6S/C111S). The three mutants and the wild-type hSOD1 (wtSOD1) were expressed in E. coli, and the soluble protein fractions and protein yields of the enzyme were evaluated. As misfolded or aggregated hSOD1 is toxic to mammalian cells [19], the toxicity and activity of mhSOD1/ C111S with the highest level of soluble expression and yields were also evaluated. All the experiments were performed to determine the mutant hSOD1 with higher soluble expression but lower toxicity and stronger activities and to find the optimal candidate for large-scale production of hSOD1.

\section{Materials and Methods}

2.1. Materials. Restriction endonucleases, Taq polymerase, T4 DNA ligase, Pfu DNA polymerase, and MiniBEST Universal Genomic DNA Extraction Kit were purchased from TaKaRa (Dalian, China). Urea, $\beta$-mercaptoethanol, and Tris$\mathrm{HCl}$ were purchased from Serva (Heidelberg, Germany). DEAE-Sepharose Fast Flow system was purchased from GE Healthcare (Wikströms, Sweden). Mouse anti-hSOD1 monoclonal antibody was purchased from Santa Cruz Biotechnology (California, USA). HRP-conjugated goat anti-mouse IgG was purchased from Zhongshan Golden Bridge Biotechnology Co. Ltd. (Beijing, China). The pET-22b (+) expression vector was purchased from Novagen (Madison, WI). The native hSOD1 gene was synthesized by Shenggong Company (Shanghai, China). All the chemicals were of analytical grade. RPMI 1640 medium and FBS were purchased from Life Technologies (Carlsbad, CA). Trypsin and MTT were purchased from Sigma (St. Louis, MO).

\subsection{Construction and Expression of Recombinant hSOD1 and} Its Mutants. The genes of hSOD1 (accession number 134611) and mutant SOD1s including mhSOD1/C6S, mhSOD1/C111S, and $\mathrm{mhSOD1/C6S/C111S}$ were synthesized by Shenggong Company.

With DNA sequencing confirmed, all the genes were digested with NdeI and SalI and cloned into pET22b (+) prokaryotic expression plasmid. Then the constructed plasmids were transformed into E. coli BL21 (DE3) competent cells. To induce SOD1 expression, positive BL21 (DE3) clones were grown overnight in $10 \mathrm{~mL}$ of LB medium supplemented with ampicillin $(100 \mu \mathrm{g} / \mathrm{mL})$ at $200 \mathrm{rpm}$ shaking and $37^{\circ} \mathrm{C}$. Afterwards, $2 \mathrm{~mL}$ of each culture was transferred to $200 \mathrm{~mL}$ of fresh LB in $500 \mathrm{~mL}$ flasks. The cultures were incubated at $37^{\circ} \mathrm{C}$ and $200 \mathrm{rpm}$ until the $\mathrm{OD}_{600}$ reached 0.5 when they were induced with $1 \mathrm{mM}$ IPTG. The cells were harvested $4 \mathrm{~h}$ later by centrifugation at $12,000 \mathrm{~g}$ for $10 \mathrm{~min}$ at $4^{\circ} \mathrm{C}$ and stored at $-20^{\circ} \mathrm{C}$. Finally, $1 \mathrm{~mL}$ of the culture was collected and analyzed by SDS-PAGE.

2.3. Analysis of the Levels of Soluble Expression of hSOD1 and mhSOD1s. Five grams of harvested cells was washed in PBS twice, resuspended in $50 \mathrm{~mL}$ of lysis buffer $(10 \mathrm{mM}$ Tris and $1 \mathrm{mM}$ EDTA, $\mathrm{pH} 8.0$ ), and sonicated on ice $(20 \mathrm{~min}$ in all, intervals of $5 \mathrm{~s}$ on/10 $\mathrm{s}$ off, power output of $300 \mathrm{~W}$ ). Then, $100 \mu \mathrm{L}$ of the lysates was centrifuged at $12,000 \mathrm{~g}$ for $30 \mathrm{~min}$ at $4^{\circ} \mathrm{C}$. The supernatant was collected as the soluble fraction, whereas the pellets (collected as insoluble fraction) were washed in PBS and resuspended in $100 \mu \mathrm{L}$ of Laemmli sample buffer (62 mM Tris-HCl, pH 6.8, 10\% glycerol, $2 \%$ SDS, $5 \% \beta$ mercaptoethanol, and $0.05 \%$ bromophenol blue). After the samples were subjected to $15 \%$ SDS-PAGE and transferred to nitrocellulose membranes $(0.22 \mu \mathrm{m}$, Invitrogen, USA), Western blot analysis was carried out. An ECL chemiluminescence system (Pierce, Rockford, USA) was used for detection. Results of the 4 independent experiments were quantified with BandScan 5.0 image analysis software.

2.4. Purification of hSOD1s. Ten grams of induced wet $E$. coli cells expressing wtSOD1, mhSOD1/C6S, mhSOD1/C111S and $\mathrm{mhSOD1/C6S/C111S} \mathrm{was} \mathrm{suspended} \mathrm{in} \mathrm{equal} \mathrm{volumes}$ of lysis buffer and disrupted by sonication. The supernatant was collected by centrifugation at $12,000 \mathrm{rpm}$ for $20 \mathrm{~min}$ at $4^{\circ} \mathrm{C}$. Then, with $1 \mathrm{mM} \mathrm{CuSO}_{4}$ added to the supernatant, the samples were heated at $70^{\circ} \mathrm{C}$ for $15 \mathrm{~min}$. After the precipitated proteins were removed by centrifugation, the supernatant was thoroughly dialyzed against a volume 30 times that of buffer $A$ (10 mM Tris- $\mathrm{HCl} \mathrm{pH} \mathrm{8.0)} \mathrm{for} 24 \mathrm{~h}$ and the insoluble particles were removed by filtering through a $0.22 \mu \mathrm{m}$ syringe filter (VWR, West Chester, PA). A DEAE-Sepharose $26 \times 200 \mathrm{~mm}$ column with $20 \mathrm{~mL}$ of column volume $(\mathrm{CV})$ was equilibrated by buffer $A$ with $5 \mathrm{CV}$. The samples were then loaded onto the column at a speed of $1 \mathrm{~mL} / \mathrm{min}$ (ÄKTA purifier, GE Healthcare) at room temperature. The column was eluted with a linear gradient of $\mathrm{NaCl}$ from 0 to $1 \mathrm{M}$ at a flow rate of $1 \mathrm{~mL} / \mathrm{min}$ with $10 \mathrm{CV}$ of buffer $B$ (buffer $A+1.0 \mathrm{M} \mathrm{NaCl}, \mathrm{pH}$ 8.0). After the purified proteins were dialyzed against $\mathrm{dd}_{2} \mathrm{O}$ for three times, they were frozen and lyophilized. Purity of the proteins was monitored by SDS-PAGE and HPLC.

2.5. Characterization of hSOD1s. The activity of the purified SOD1 proteins was determined as previously described [20]. In brief, $150 \mu \mathrm{L}$ of $4.5 \mathrm{mM}$ pyrogallol was added to $4.35 \mathrm{~mL}$ Tris- $\mathrm{HCl}$ buffer (0.1 M Tris, $1 \mathrm{mM}$ EDTA-2Na, and $\mathrm{pH} 8.2$ ) for autoxidation, and the absorbance was assessed at a wavelength of $325 \mathrm{~nm}\left(\mathrm{OD}_{325}\right)$ with the autoxidation rate 
modulated to approximately $0.06 \mathrm{OD} \mathrm{min}^{-1}$. SOD1 activity was measured by monitoring the change in $\mathrm{OD}_{325}$ resulting from the inclusion of $20 \mu \mathrm{L}$ of the sample in the $4.35 \mathrm{~mL}$ Tris-HCl buffer solution prior to the addition of pyrogallol. Generally, a unit of the enzyme is defined as the amount of the enzyme which inhibits the pyrogallol autoxidation rate by $50 \%$ per min.

The amounts of the metals copper and zinc were measured using a novAA300 atomic absorption spectrometer (Analytik Jena AG, Germany) [21].

2.6. Analysis of hSOD1s Toxicity. Converging evidence indicates that misfolded or aggregated hSOD1 is toxic to mammalian cells through several ways [19, 22-24]. To evaluate the toxicity of the wtSOD1 and mhSOD1/C111S, Baby Hamster Kidney (BHK) cells suspended in RPMI 1640 medium (8 $\times 10^{3}$ cells in $200 \mu \mathrm{L}$ ) were seeded in 96-well culture plates in triplicate and treated with serially diluted SOD1s for 1-5 days. Bovine serum albumin (BSA) was used as the negative control. The cell numbers were measured by Cell Counting Kit-8 (BioTek Inc., VA) according to the manufacturer's instruction. The cell proliferation rate was calculated from the cell numbers and normalized to BSA-treated cells.

To further investigate the toxicity of endogenous mhSOD1/ C111S, both wtSOD1 and mhSOD1/C111S genes were cloned into pcDNA3 (Invitrogen) plasmid and transfected into BHK cells with Lipofectamine 2000 according to standard instructions. Cell growth rate was calculated at 24 and $48 \mathrm{~h}$ after transfection. To evaluate the solubility and aggregation of SOD1s, BHK cells were collected and the supernatant (soluble fraction) and the pellet (insoluble fraction) samples were prepared according to a previously described method [18]. Finally, soluble and insoluble SOD1s expressed in BHK cells were detected by Western blot.

2.7. Whitening Effects of hSOD1s. The whitening effects of wild-type and mutant SOD1s were measured by their modulation of melanocytic proliferation and tyrosinase activity according to standard methods.

To detect the effects of SOD1s on melanocytic proliferation, 3-4 $\times 10^{3}$ mouse skin melanoma B16 cells were seeded in 96-well plates in triplicate and cultured for $48 \mathrm{~h}$ at $37^{\circ} \mathrm{C}$ under $5 \% \mathrm{CO}_{2}$ in humid air. Next, SOD1s were added and cell proliferation was analyzed by MTT assay as previously described [25]. In brief, the cells were incubated for 1, 2, or 3 days, respectively, and $20 \mu \mathrm{L}$ of MTT $(0.5 \mathrm{mg} / \mathrm{mL})$ was added. Then, the plates were centrifuged (2000 rpm for $10 \mathrm{~min}$ ) $4 \mathrm{~h}$ later and the supernatants were discarded. Finally, $100 \mu \mathrm{L}$ of DMSO was added and the solubilized MTT was measured at $570 \mathrm{~nm}$ using a Bio-Rad plate reader and the cell growth rate was calculated with the formula: Growth rate $(\%)=\mathrm{OD}$ sample/OD control $\times 100 \%$.

The in vitro tyrosinase inhibition assays were performed according to the method described previously [26, 27]. In brief, $30 \mu \mathrm{L}$ of mushroom tyrosinase (final concentration $6.67 \mu \mathrm{g} / \mathrm{mL}$ ) was preincubated with serially diluted wtSOD1 and $\mathrm{mhSOD} 1 / \mathrm{C} 111 \mathrm{~S}(1.25,2.5,3.75,5.0,7.5$, and $10.0 \mathrm{mg} / \mathrm{mL})$ in $50 \mathrm{mM} \mathrm{NaHPO}-\mathrm{NaH}_{2} \mathrm{PO}_{4}$ buffer (pH 6.8) for $10 \mathrm{~min}$ at $30^{\circ} \mathrm{C}$. The substrate L-DOPA (dihydroxyphenylalanine,
$0.5 \mathrm{mM}$ ) was added to the mixture and formation of the Ldopaquinone was continuously monitored by assaying the change in absorbance at $475 \mathrm{~nm}$ for $1 \mathrm{~min}$. The activity was expressed as the sample concentration, which inhibited 50\% of the enzyme activity (IC50). The percentage of inhibition of tyrosinase was calculated as follows: Inhibition rate $(\%)=$ $(A-B) / A \times 100 \%$, where $A$ and $B$ represent the absorbance values for the blank and for the samples, respectively.

The in vivo tyrosinase inhibition was assayed by using B16 cells. In brief, $3-4 \times 10^{3}$ cells were seeded in 96 -well plates and cultured for $24 \mathrm{~h}$ at $37^{\circ} \mathrm{C}$. Then, wtSOD1 and mhSOD1/C111S serially diluted with RPMI $1640(0.0039,0.0156,0.0625,0.25$, and $1 \mathrm{mg} / \mathrm{mL}$ ) were added and the cells were cultured for another $48 \mathrm{~h}$. RPMI 1640 medium was used as the negative control. After being washed with cold PBS twice, cells were lysed in $90 \mu \mathrm{L} 1 \%$ Triton X-100 (in PBS) by freezing. Finally, $10 \mu \mathrm{L} \mathrm{L}$-Dopa $(0.1 \%)$ was added and incubated at $37^{\circ} \mathrm{C}$ for $1 \mathrm{~h}$ and the absorbance at $475 \mathrm{~nm}$ was assayed to calculate the inhibition percentage of tyrosinase as follows: Inhibition rate $(\%)=(1-($ OD sample $\div$ OD control $)) \times 100 \%$.

2.8. Determination of the Antiradiation Activity of hSOD1s. One of the most important functions of hSOD1 is that it can resist radiation. To test the antiradiation activities of SOD1s, wtSOD1 and mhSOD1/C111S were used to treat BHK cells under ultraviolet (UV) radiation and the protective effects of SOD1s on cell viability and DNA damage were measured.

To evaluate the protective effects of wtSOD1 and mhSOD1/ C111S on UV irradiation, about $2 \times 10^{5}$ BHK cells in $200 \mu \mathrm{L}$ medium were seeded in 48 -well plates and cultured for $24 \mathrm{~h}$. Then, the cells were washed with PBS and incubated in $100 \mu \mathrm{L}$ of fresh RPMI 1640 medium containing 1\% FBS. Afterwards, wtSOD1 and mhSOD1/C111S diluted with RPMI 1640 medium $(0.0039,0.0078,0.0156,0.0312$, and $0.0625 \mathrm{mg} / \mathrm{mL})$ were added after PBS washing. Kojic acid and arbutin were used as positive controls. Cells that received radiation or none in the absence of both wtSOD1 and mhSOD1/C111S were used as normalization controls. The protection ratio was calculated with the formula: (OD sample - OD radiative control)/(OD nonradiative control - OD radiative control) $\times 100 \%$.

For DNA damage analysis, BHK cells were seeded in 6-well plates and treated as described above. After being irradiated with UV, the cells were cultured in a fresh medium for $12 \mathrm{~h}$ and washed with PBS once. Then, the cells were incubated with fresh PBS for $10 \mathrm{~min}$ and harvested by centrifugation. Finally, genomic DNA was extracted and the DNA damage was analyzed by agarose gel electrophoresis.

All the experiments were repeated 4 times and the medium without SOD1 was used as control groups.

2.9. Statistical Analysis. The statistical significance of the differences was evaluated using Student's two-tailed $t$-test. Differences were considered significant when $P<0.05$.

\section{Results and Discussion}

In the previous work, for large-scale production of recombinant hSOD1, wtSOD1 was expressed in E. coli, in which soluble wtSOD 1 accounted for approximately $36 \%$ of the total 


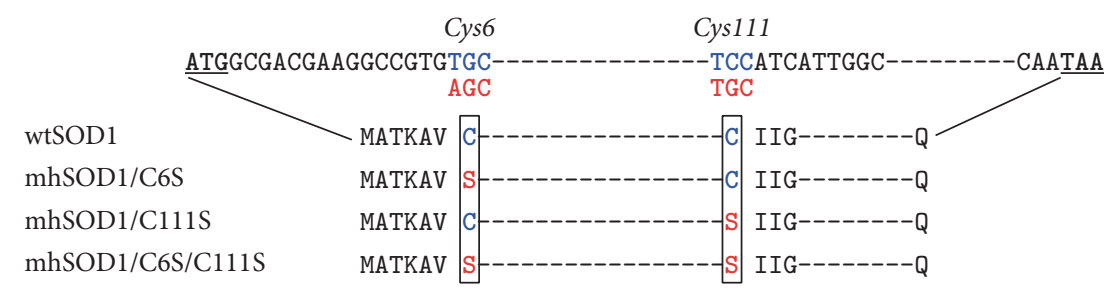

(a)

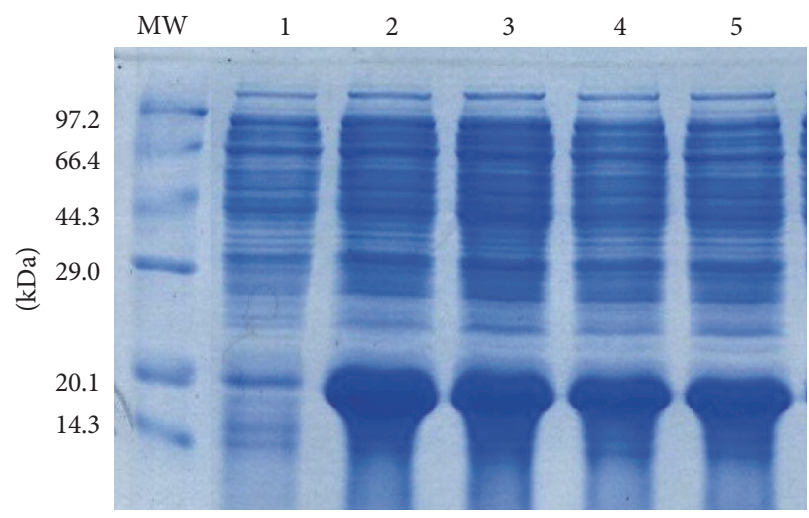

(b)

FIGURE 1: Construction and expression levels of wtSOD1 and its mutants in E. coli. (a) Schematic diagram of the molecular structure of the wild-type and mutant SODs constructed. Blue color indicates the wild-type DNA or amino acid sequences and red color indicates the mutant ones. (b) Expression of wtSOD1 and its mutants by SDS-PAGE analysis. Lane MW: molecular weight standard ladder (kDa). Lane 1: whole cell lysate of wtSOD1-expressing cells before induction. Lanes 2-5: whole cell lysate of cells expressing wtSOD1 (lane 2), mhSOD1/C6S (lane 3), mhSOD1/C111S (lane 4), and mhSOD1/C6S/C111S (lane 5) after induction.

expressed protein. Then, wtSOD1 was purified from both the intracellular inclusions and the cell lysate supernatant. The specific activity of the protein purified from the cell lysate supernatant was approximately $6000 \mathrm{IU} / \mathrm{mg}$, which was 3 times higher than that of the enzyme purified from the inclusion bodies (unpublished data). In view of these results, we expected that increasing the soluble expression levels of SOD1 in E. coli might effectively improve the yield of hSOD1. In this study, a method for substituting the free cysteines in SDO1 was used to increase the soluble expression and the yield of hSOD1 in E. coli.

3.1. Construction and Expression of Recombinant wtSOD1 and Its Mutants. All the DNA fragments encoding wtSOD1 and three mutants, in which Cys6 (mhSOD1/C6S), Cys111 (mhSOD1/C111S), or both Cys6 and Cys111 (mhSOD1/C6S/ C111S) were substituted with serine, were synthesized by Shenggong Company (Figure 1(a)). The successful insertion of DNA fragments encoding wtSOD1 or its mutants into pET-22b (+) bacterial expression plasmids was confirmed by digestion with NdeI and SalI and DNA sequencing (data not shown). Then, wtSOD1 and its mutants were successfully induced in E. coli BL21 (DE3) when the transformed bacteria were treated by $1 \mathrm{mM}$ IPTG (Figure 1(b)). Molecular weight of the expressed proteins was approximately $20.1 \mathrm{kDa}$, which is consistent with the results obtained by Hartman et al. [28] (Figure 1(b)). The recombinant proteins represented approximately $55 \%$ of the total bacterial protein, as is determined by densitometric scanning.
3.2. Comparison of Soluble Expression Levels of Recombinant SOD1s. Western blot results have confirmed that a hSOD1 monoclonal antibody could effectively detect the expressed wtSOD1 and all of its mutants (Figures 2(a) and 2(b)). The protein expressions in the soluble and insoluble fractions were compared. Soluble wtSOD1 accounted for approximately $36.1 \%$ of the total expressed SOD1 (Figure 2(c)). The single substitution of Cys6 (mhSOD1/C6S) decreased soluble SOD1 expression remarkably from $36.1 \%$ to $20.8 \%$ whereas the single substitution of Cys111 (mhSOD1/C111S) increased soluble expression significantly from $36 \%$ to $84.9 \%$. No significant difference was found between the soluble protein expression levels of the double mutant mhSOD1/C6SC111S and that of mhSOD1/C111S.

These results suggest that Cysl11 is a mediator essential to soluble expression. The observed decrease in the expression of soluble protein when Cys6 was substituted suggests that during the protein folding process, the inner free cysteine may prevent the formation of an unfavorable disulfide bond between Cys111 and either Cys57 or Cys148. This is supported by the expression level of the soluble protein, mhSOD1/C6S/ C111S, which is similar to that of mhSOD1/C111S. The expression levels of the two soluble proteins are significantly higher than that of mhSOD1/C6S (Figure 2).

3.3. Comparison of the Protein Yield and Activity of Recombinant wtSOD1 with Its Mutants. The lytic supernatants of the cell cultures expressing recombinant wtSOD1, mhSOD1/C6S, $\mathrm{mhSOD} 1 / \mathrm{C} 111 \mathrm{~S}$, and $\mathrm{mhSOD} 1 / \mathrm{C} 6 \mathrm{~S} / \mathrm{C} 111 \mathrm{~S}$ were purified with 


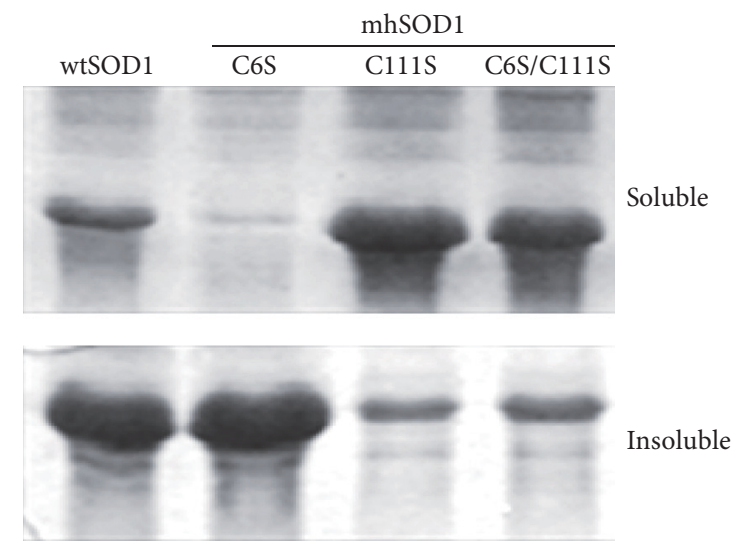

(a)

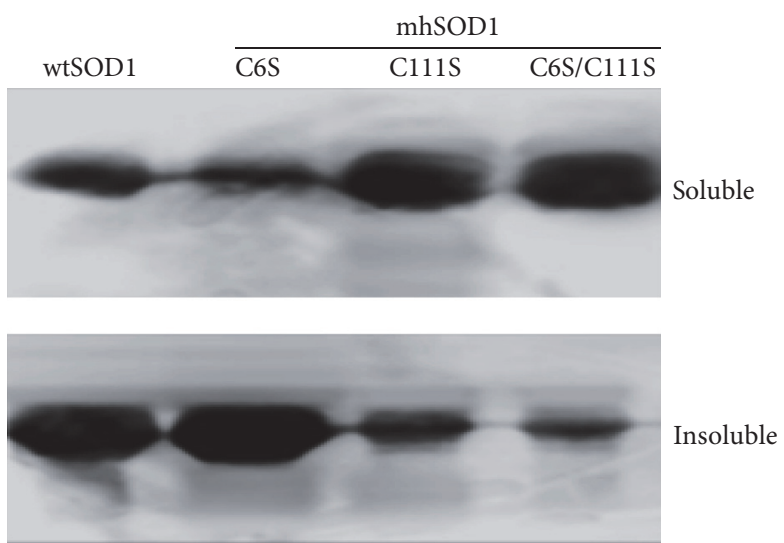

(b)

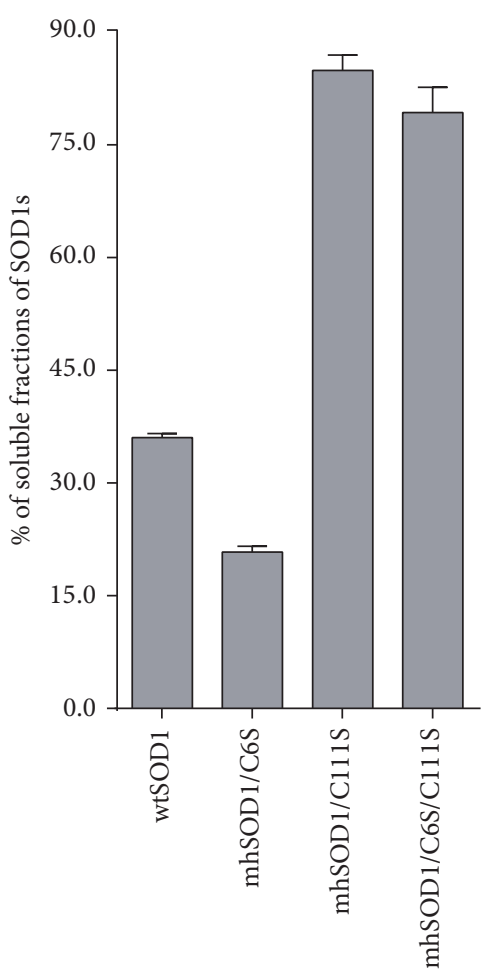

(c)

FIGURE 2: Effects of cysteine mutations on the solubility of hSOD1 expressed in E. coli. (a) SDS-PAGE of soluble and insoluble protein fractions. (b) Western blotting of soluble proteins. Proteins on the SDS-PAGE gel were transferred to membranes, blotted with an anti-SOD1 antibody to determine the relative percentage of the soluble fractions. The lanes contain wtSOD1 or three mutants as indicated. (c) Histogram of the distribution of the soluble fractions of SOD1 in E. coli as determined by 4 independent Western blot experiments.

DEAE-Sepharose Fast Flow columns. The purity of the isolated proteins was above $95 \%$ in all the cases, as confirmed by SDS-PAGE (Figure 3(a)) and HPLC (Figures 3(b)-3(e)). The activity yields of wtSOD1, mhSOD1/C6S, mhSOD1/C111S, and mhSOD1/C6S/C111S purified per gram of wet E. coli cells showed the following progression: $\mathrm{mhSOD} 1 / \mathrm{C} 111 \mathrm{~S}$ and mhSOD1/C6S/C111S > wtSOD1 > mhSOD1/C6S (wtSOD1, 6.2 $\times 10^{4} \pm 2.1 \times 10^{3} \mathrm{IU} ; \mathrm{mhSOD} 1 / \mathrm{C} 6 \mathrm{~S}, 1.6 \times 10^{4} \pm 1.5 \times 10^{3} \mathrm{IU}$; $\mathrm{mhSOD1} / \mathrm{C} 111 \mathrm{~S}, 2.02 \times 10^{5} \pm 3.5 \times 10^{3} \mathrm{IU}$; mhSOD1/C6S/C111S, $\left.1.98 \times 10^{5} \pm 5.3 \times 10^{3} \mathrm{IU}\right)$. All these results collectively verified our hypothesis that an increase in the soluble expression levels may increase hSOD1 yields (Table 1). For this reason, mhSOD1/C111S was selected for our subsequent experiments to investigate the toxicity and activity of mhSOD1s, whereas wtSOD1 was used as the control.

Considering the presence of copper and zinc ions in the active site of SOD1 enzymes is critical for their activity, both copper and zinc contents of the purified proteins were determined. The results from the atomic absorption measurements of the purified proteins were showed in Table 2 and it showed that mhSOD1/C111S increased the metallization considerably. 


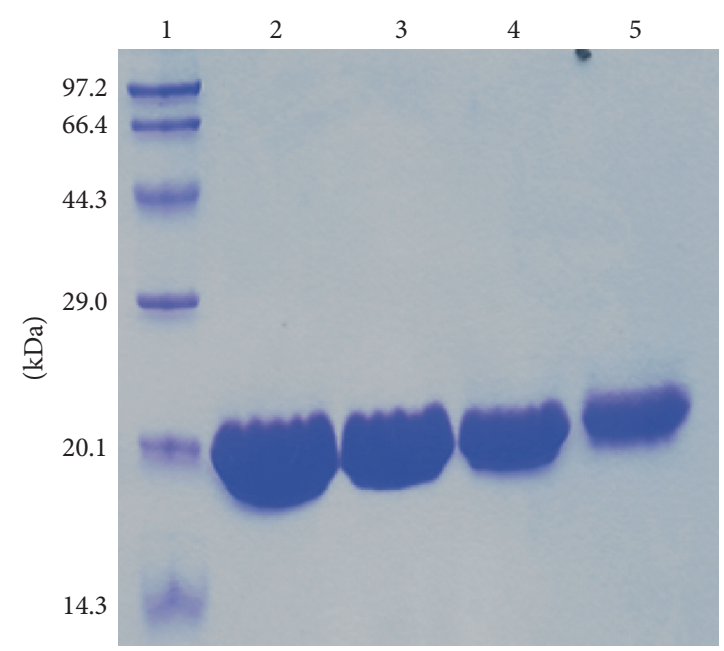

(a)

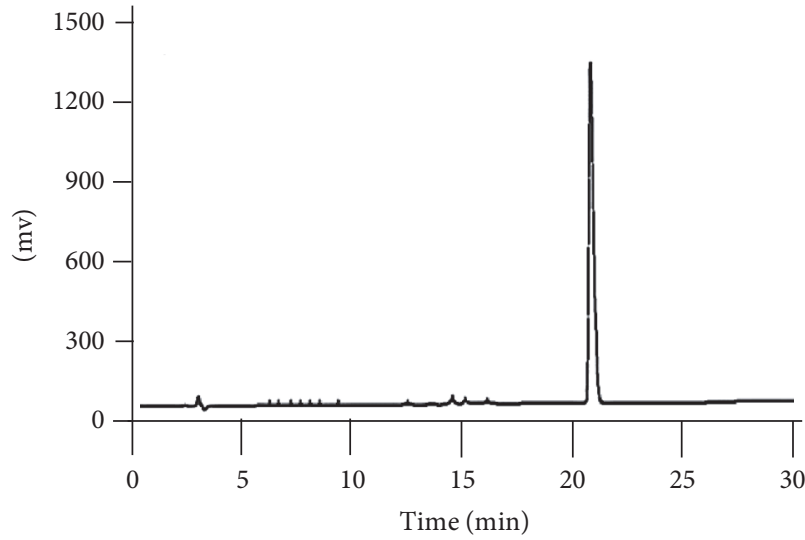

(b)

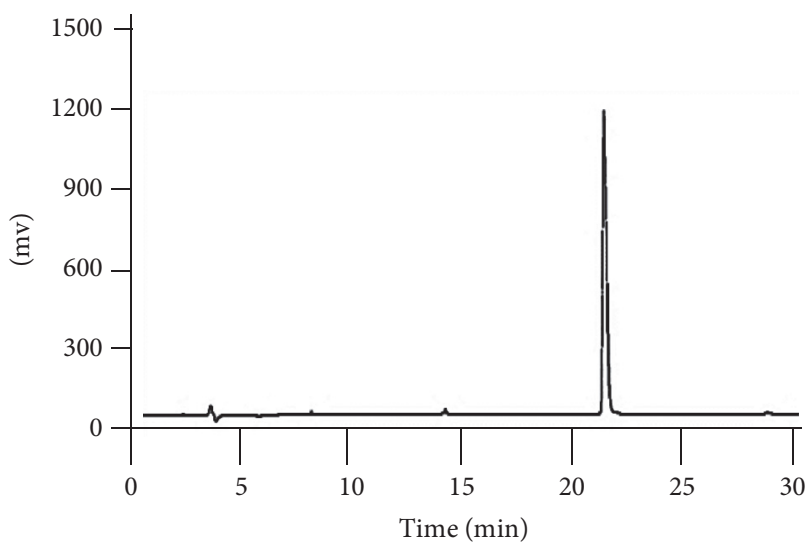

(d)

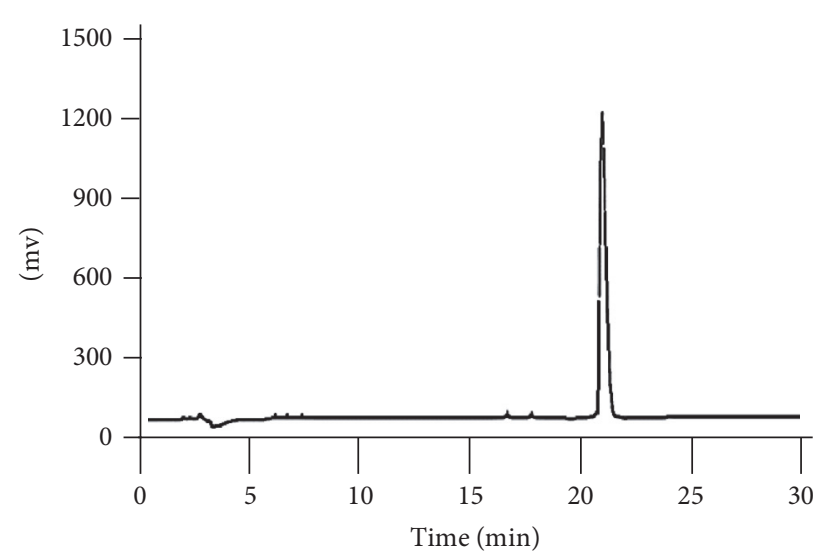

(c)

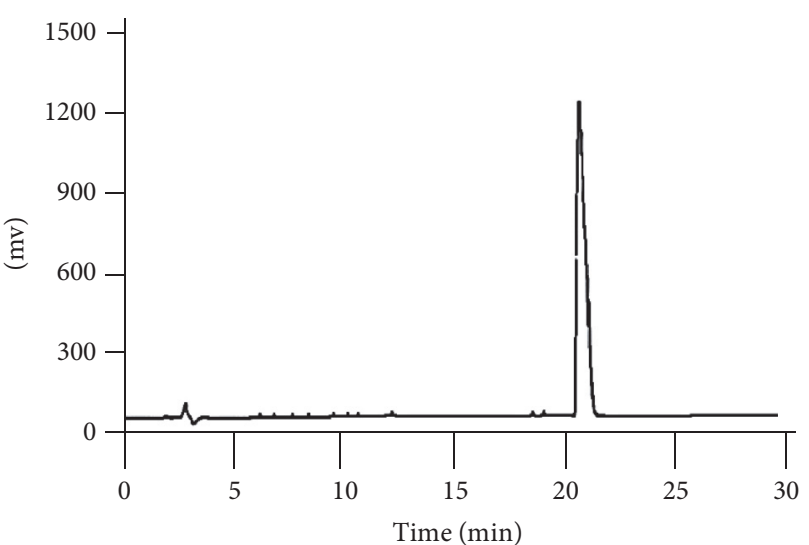

(e)

FIgURe 3: Purity analysis of SOD1s by SDS-PAGE and HPLC. (a) Purity of SOD1s was analyzed by SDS-PAGE. Lane 1: molecular weight standard ladder (kDa). Lanes 2-5: purified wtSOD1 (lane 2), mhSOD1/C6S (lane 3), mhSOD1/C111S (lane 4), and mhSOD1/C6S/C111S (lane 5). (b) HPLC analysis of purified wtSOD1 (a), mhSOD1/C6S (b), mhSOD1/C111S (c), and mhSOD1/C6S/C111S (d). A reverse-phase column was used for HPLC (Beckman, $250 \times 5.0 \mathrm{~mm}$ ID, Kromasil C18, Akzo Nobel Co. Ltd. (Sweden)). $5 \mu \mathrm{g}$ samples in PBS were injected into the column and then eluted by linear gradient: $100 \%$ solution A (water, $5 \%$ acetone, and $0.1 \%$ TFA) to $40 \%$ solution B (acetone, $0.1 \%$ TFA) over 40 minutes with a flow rate of $1.0 \mathrm{~mL} / \mathrm{min}$. The eluted fractions were analyzed at $280 \mathrm{~nm}$, and the purity of the proteins was calculated as a percentage of the total peak area. 
TABLE 1: Ratios of mhSOD1s to wtSOD1 in soluble expression and yields.

\begin{tabular}{lcccc}
\hline & wtSOD1 & mhSOD1/C6S & mhSOD1/C111S & mhSOD1/C6S/C111S \\
\hline Soluble expression & 1.00 & $0.58 \pm 0.02 \downarrow$ & $2.35 \pm 0.07 \uparrow$ & $2.20 \pm 0.10 \uparrow$ \\
Activity yield & 1.00 & $0.26 \pm 0.02 \downarrow$ & $3.25 \pm 0.07 \uparrow$ & $3.19 \pm 0.04 \uparrow$ \\
\hline
\end{tabular}

The ratios of mhSOD1s to wtSOD1 in soluble expression and yields were calculated. $\uparrow$ presents increase and $\downarrow$ presents decrease when compared with the wtSOD1.
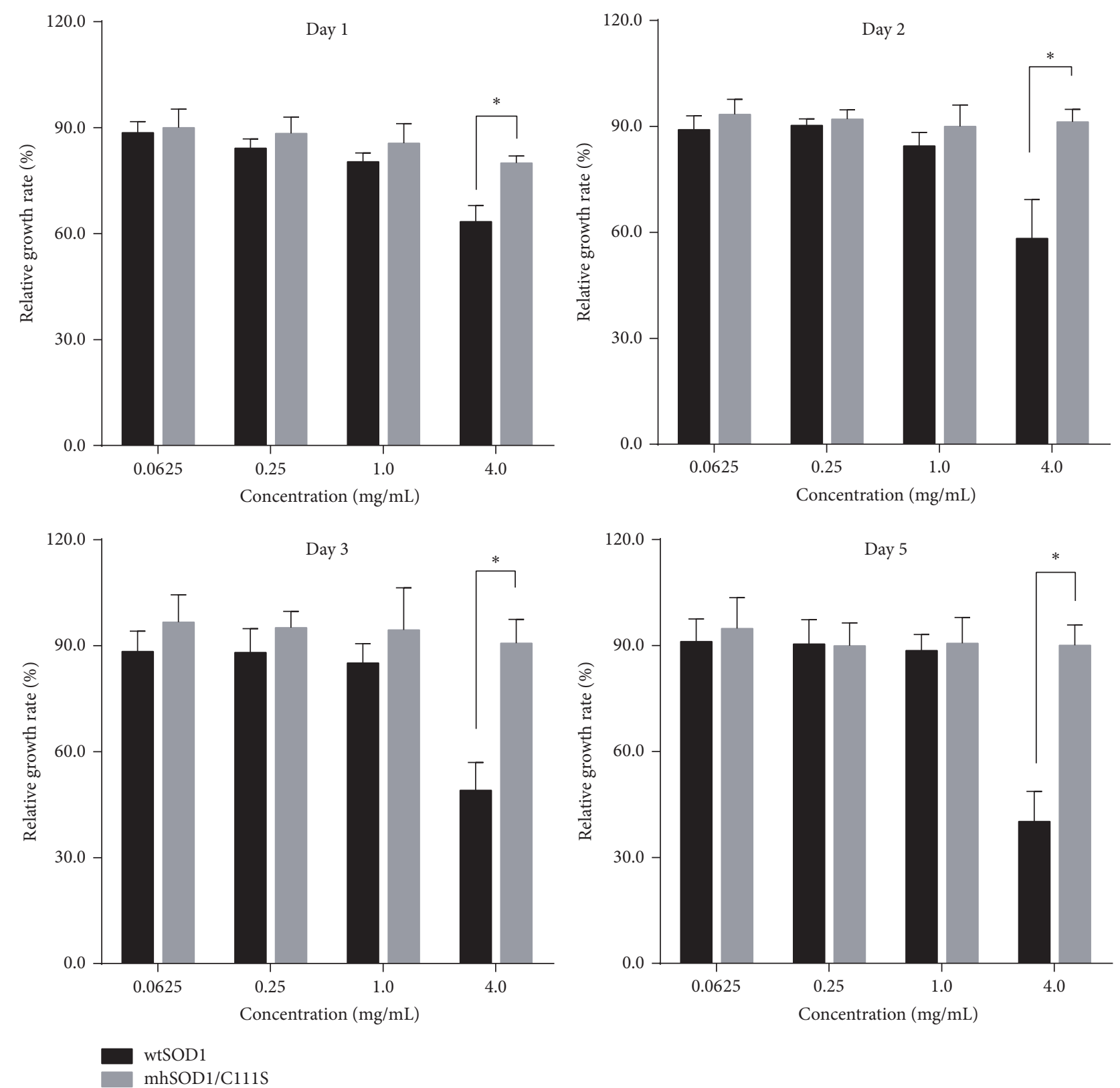

FIGURE 4: Toxicity of wtSOD1 and mhSOD1/C111S to BHK cells. BHK cells were treated with recombinant SOD1s, which were serially diluted with RPMI 1640 medium, for 1-5 days and cell proliferation was assessed by CCK- 8 method. BSA was used as the negative control. ${ }^{*} P<0.05$ compared between indicated groups. Data are representative of three experiments.

3.4. Toxicity of Recombinant SOD1s. To determine whether the cysteine substitution can affect the toxicity of recombinant SOD1, wtSOD1 and mhSOD1/C111S were used to treat BHK cells and cell proliferation was assessed by CCK-8 solution at indicated time points. Bovine serum albumin
(BSA) was used as the negative control in which the growth rate of the cells was set as 100\%. As shown in Figure 4, neither wtSOD1 nor mhSOD1/C111S had hardly any impact on cell proliferation when the dosage was low $(0.0625-1.0 \mathrm{mg} / \mathrm{mL})$. As to higher dosage, $4.0 \mathrm{mg} / \mathrm{mL}$ wtSOD1 is apparently toxic 
TABLE 2: Atomic absorption measurements of $\mathrm{Cu}$ and $\mathrm{Zn}$ contents in SOD1 proteins.

\begin{tabular}{|c|c|c|c|c|}
\hline \multirow{2}{*}{ SOD protein } & \multicolumn{2}{|c|}{$\mathrm{Cu}$ content } & \multicolumn{2}{|c|}{ Zn content } \\
\hline & $\mu \mathrm{g} / \mathrm{mg}$ & $\mathrm{Cu} /$ subunit $^{\mathrm{a}}$ & $\mu \mathrm{g} / \mathrm{mg}$ & $\mathrm{Zn} /$ subunit $^{\mathrm{a}}$ \\
\hline wtSOD1 & 4.346 & 1.37 & 2.787 & 0.85 \\
\hline mhSOD1/C111S & 5.322 & 1.67 & 3.822 & 1.16 \\
\hline
\end{tabular}

${ }^{\mathrm{a}}$ The molecular weight of wtSOD1 and mhSOD1/C111S is calculated as $20 \mathrm{kDa}$.

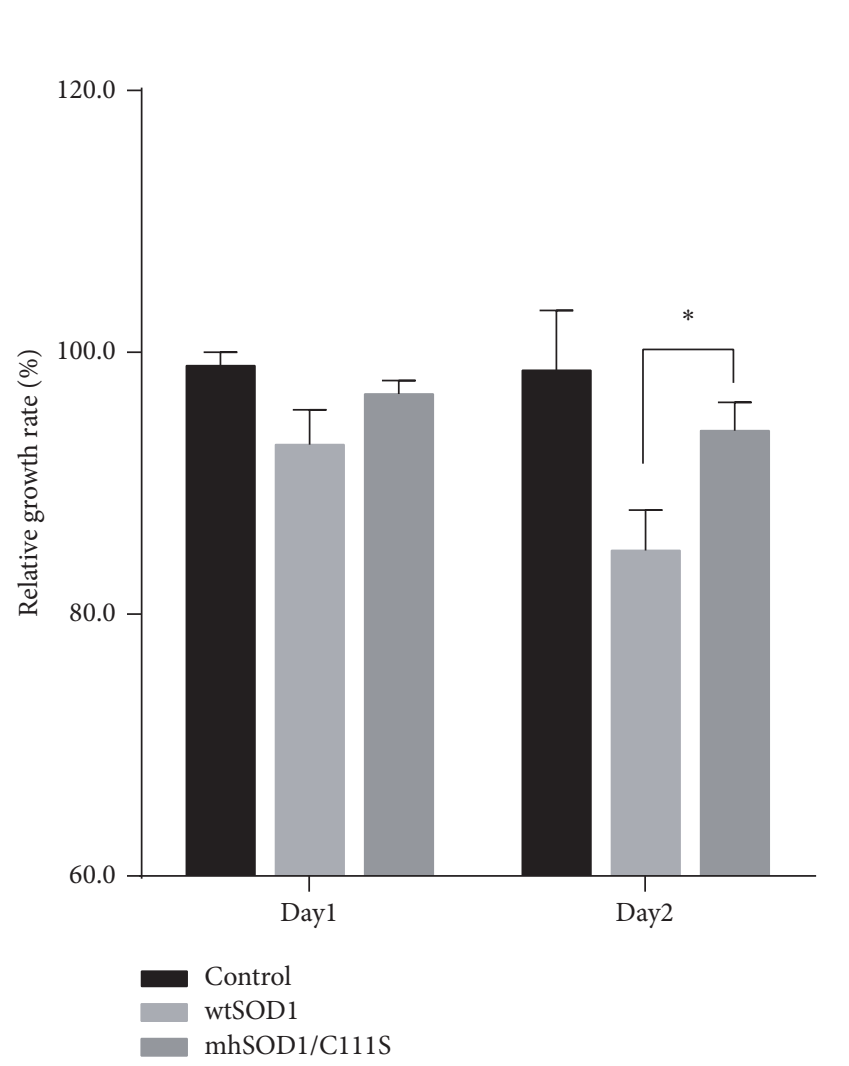

(a)

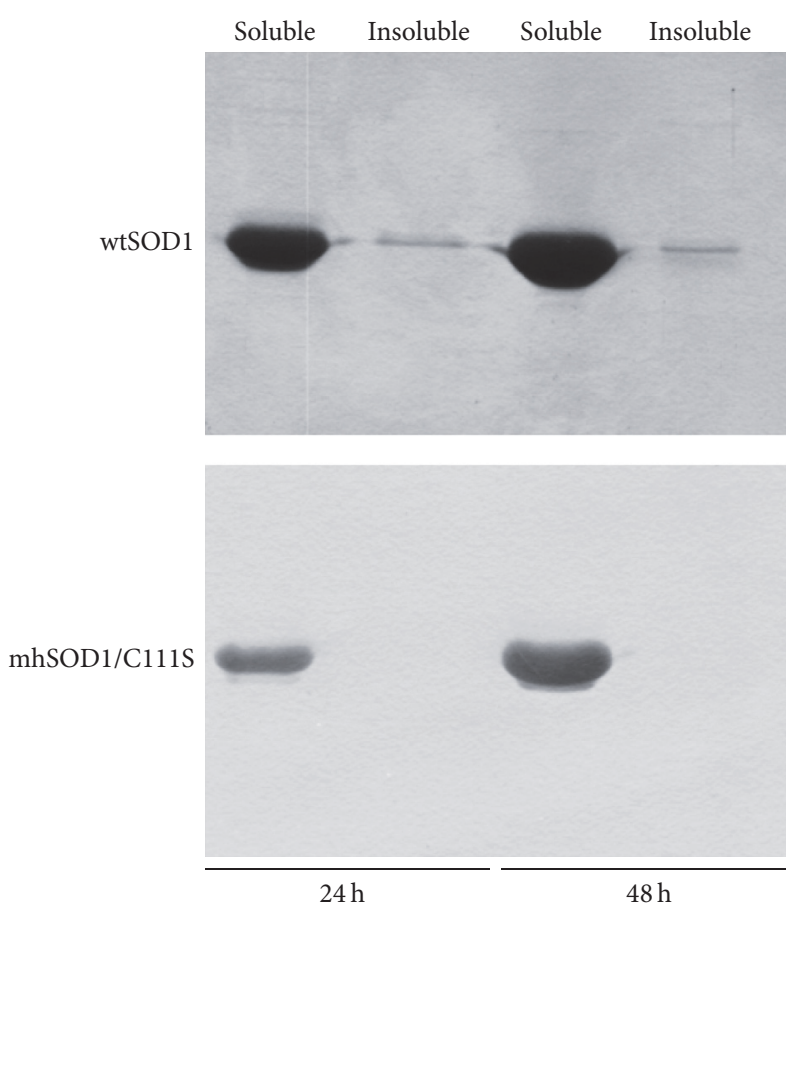

(b)

FIgURE 5: Toxicity of wtSOD1 and mhSOD1/C111S to BHK cells in vivo. BHK cells were transfected with recombinant plasmids pcDNA3wtSOD1 and pcDNA3-mhSOD1/C111S by using Lipofectamine 2000. The empty plasmid pcDNA3 was used as the negative control. Cell proliferation was assessed by CCK- 8 method and SOD1s expression as soluble and insoluble forms was detected by Western blot $24 \mathrm{~h}$ and $48 \mathrm{~h}$ after transfection. ${ }^{*} \mathrm{P}<0.05$ compared between indicated groups. Data are representative of three experiments.

to $\mathrm{BHK}$ cells and the relative growth rate of the cells is reduced by $50-60 \%$, whereas the toxicity of $\mathrm{mhSOD} 1 / \mathrm{C} 111 \mathrm{~S}$ is significantly lower than that of wtSOD1 $(P<0.05)$ since the cells treated with $\mathrm{mhSOD} 1 / \mathrm{C} 111 \mathrm{~S}$ for $1-5$ days maintained their normal proliferation. These data indicate that to $\mathrm{BHK}$ cells mhSOD1/C111S is safer than wtSOD1.

To study the possible toxicity of mhSOD1/C111S in mammalian cells, the ability of mhSOD1/C111S to affect cell proliferation and SOD1 solubility was tested in a cell transfection assay. As shown in Figure 5(a), wtSOD1 transfection remarkably inhibited whereas mhSOD1/C111S has no apparent effect on BHK cell proliferation. As to SOD1 solubility, only a subtle aggregation was detected in wtSOD1-transfected BHK cells, but almost all mhSOD1/C111S is accumulated in the soluble form and no aggregation was found (Figure 5(b)). Both wtSOD1 and mhSOD1/C111S are more soluble in mammalian cells than E. coli, and it is most probably because of a more powerful chaperone system and more effective redox environment in mammalian cells.

3.5. Whitening Effects of SOD1. To investigate the whitening effects of SOD1s, we first assessed the effects of SOD1s on B16 melanocyte proliferation. Results showed that both wtSOD1 and $\mathrm{mhSOD1/C111S}$ apparently inhibit B16 cell proliferation at higher dosages $(0.25-1 \mathrm{mg} / \mathrm{mL}$ ) on days 2 and 3 (Figure 6(a)). Next, we detected the inhibitory effects of SOD1s on tyrosinase. As shown in Figures 6(b) and 6(c), both wtSOD1 and mhSOD1/C111S apparently inhibited tyrosinase activity in vitro and in vivo. Interestingly, the effects of mhSOD1/C111S are stronger than that of wtSOD1 in the cell-free in vitro system and the in vivo system. We conjecture that this is probably due to the fact that the conformation or half-life of 

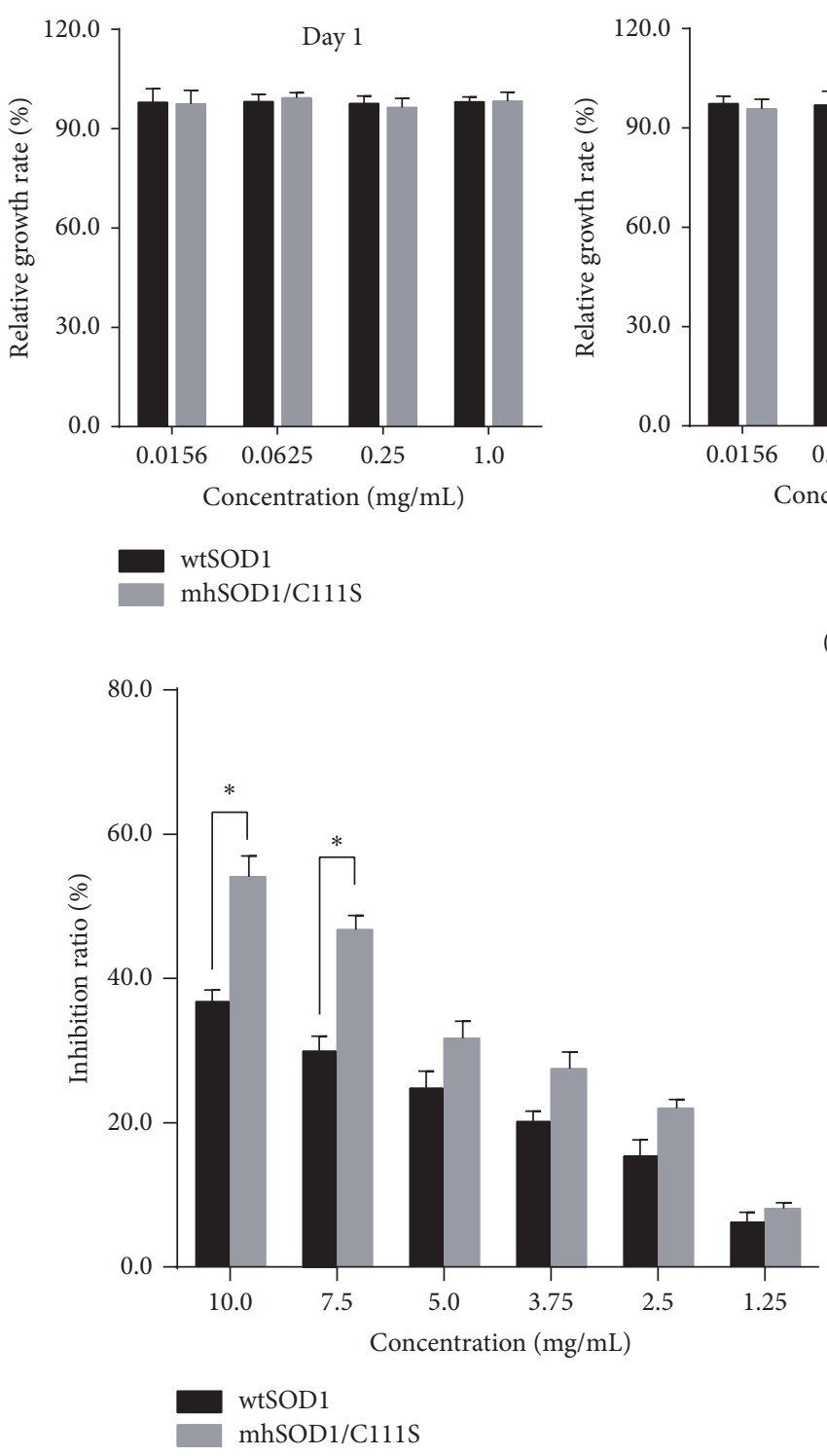

(b)
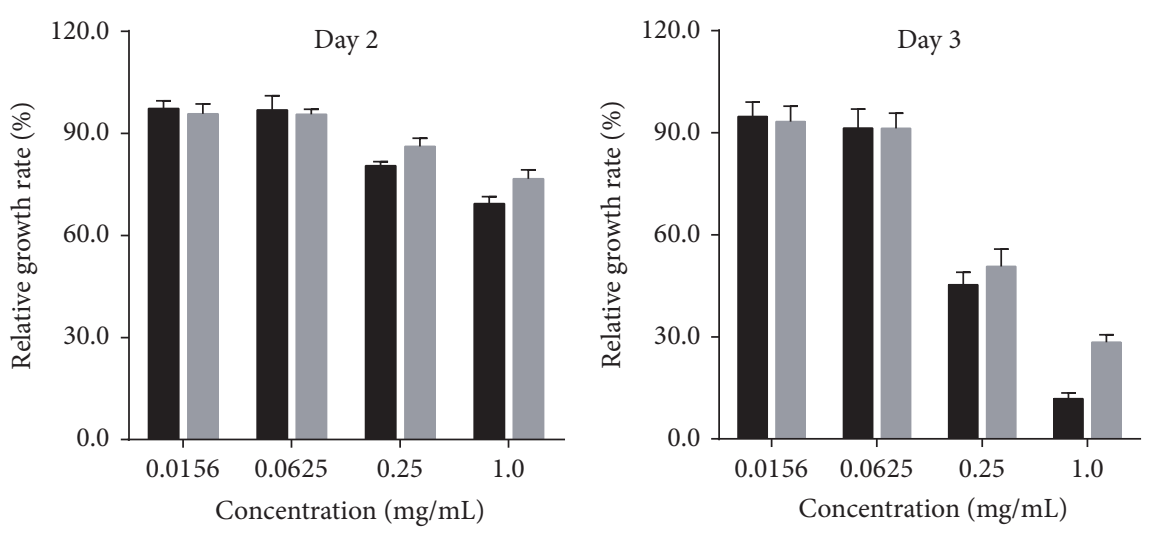

(a)

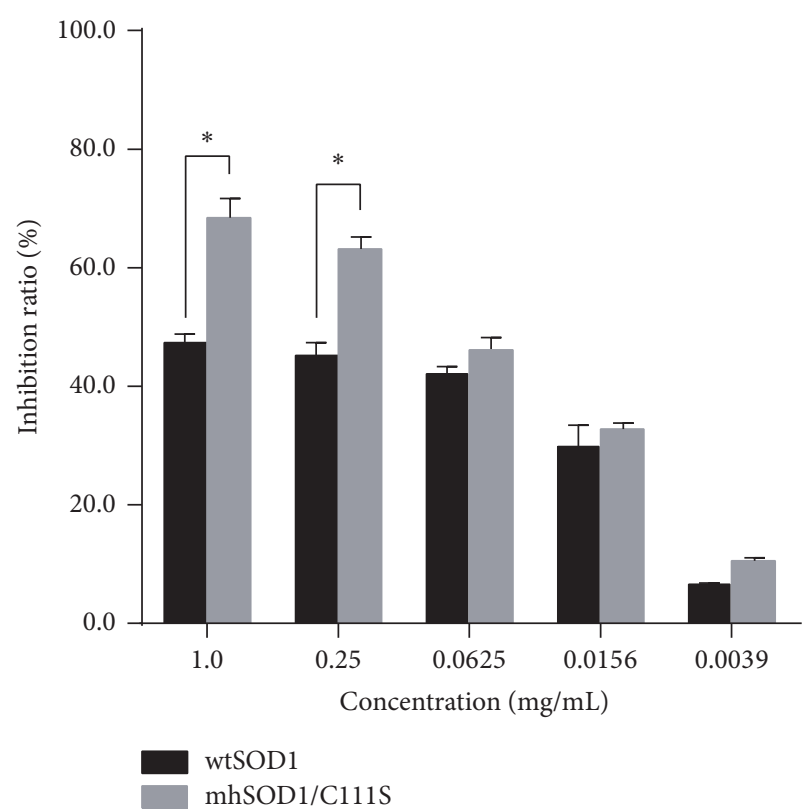

(c)

FIGURE 6: Whitening effects of wtSOD1 and mhSOD1/C111S. (a) B16 cells were treated with both wtSOD1 and mhSOD1/C111S diluted with RPMI 1640 medium and cell proliferation was analyzed by MTT assay. ((b) and (c)) The inhibition of mushroom tyrosinase activity by wtSOD1 and mhSOD1/C111S was measured in vitro (b) and in vivo (c). ${ }^{*} P<0.05$ compared between indicated groups.

mhSOD1/C111S is longer than that of wtSOD1. The detailed mechanism of this is still under investigation. In addition, we found that the effects of both wtSOD1 and mhSOD1/C111S on melanocytic proliferation and mushroom tyrosinase are not only time-dependent but also dose-dependent.

3.6. Antiradiation Activity of SOD1. To test the antiradiation activity of SOD1s, wtSOD1 and mhSOD1/C111S were used to treat $\mathrm{BHK}$ cells under UV radiation and the protective effects of SOD1s on cell viability and DNA damage were measured. Figure $7(\mathrm{a})$ shows that $\mathrm{mhSOD} 1 / \mathrm{Cl11S}$ has a stronger antiradiation effect than wtSOD1 $(P<0.05)$. The protective effect of mhSOD1/C111S on UV-treated BHK cells is even slightly better than that of kojic acid and arbutin.
When higher dosages $(0.0625$ and $0.0312 \mathrm{mg} / \mathrm{mL})$ are given, significant differences were found between mhSOD1/C111Sand wtSOD1-treated cells $(P<0.001$ and $P<0.01$, resp. $)$ with the former having a stronger activity (Figure $7(\mathrm{~b})$ ). We further detected DNA damage in UV-radiated BHK cells. As shown in Figure 7(c), both wtSOD1 and mhSOD1/C111S treatments effectively prevented DNA damage in BHK cells when compared with UV-radiated cells.

\section{Conclusions}

As a soluble cytoplasmic and mitochondrial intermembrane superoxide dismutase, hSOD1 can prevent damage to cell components caused by free radicals derived from aerobic 


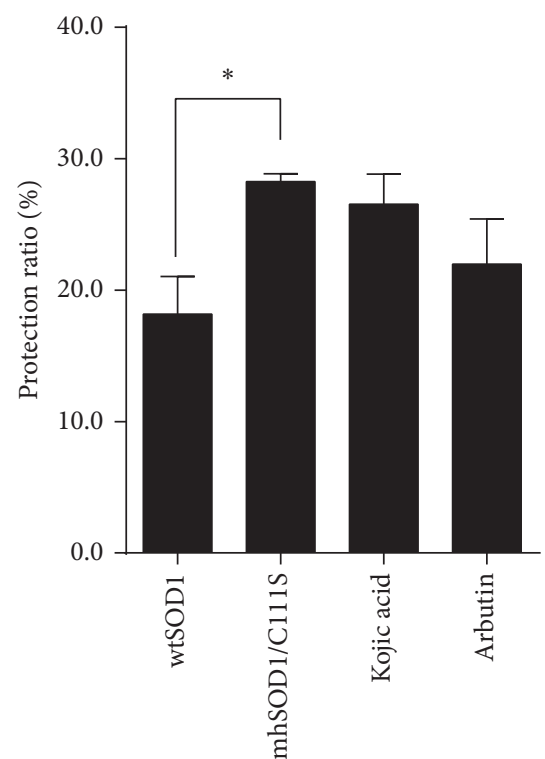

(a)

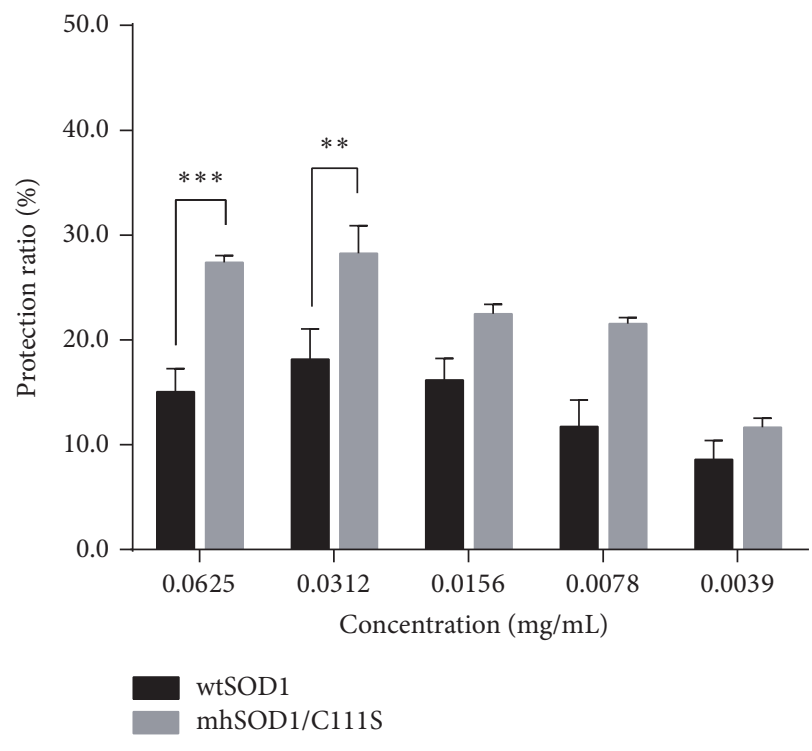

(b)

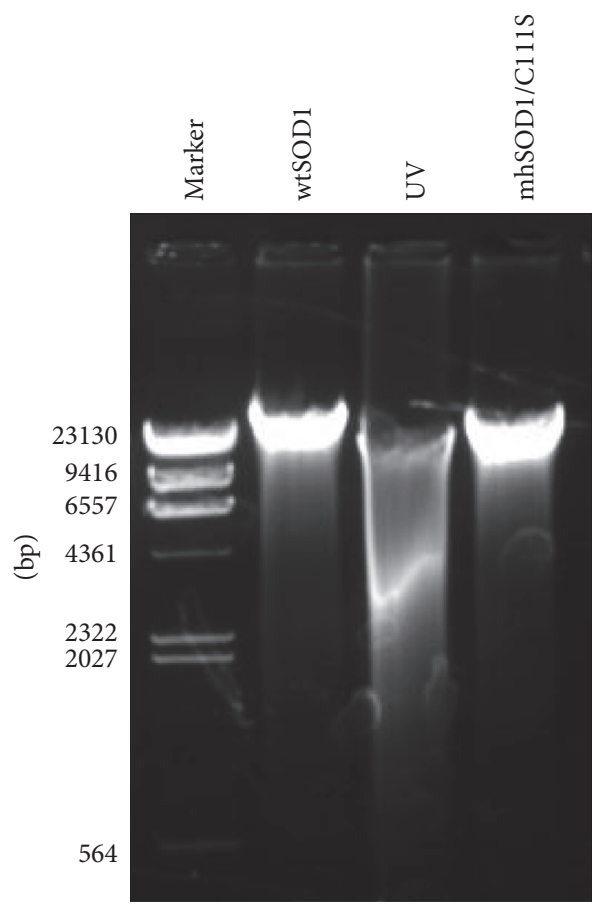

(c)

FIGURE 7: Antiradiation effects of SOD1s. (a) BHK cells were treated with wtSOD1 and mhSOD1/C111S and cell proliferation was analyzed by MTT assay. Kojic acid and arbutin were used as positive controls. (b) BHK cells were treated with serially diluted wtSOD1 and mhSOD1/C111S and cell proliferation was analyzed by MTT assay. ${ }^{*} P<0.05 ;{ }^{* *} P<0.01 ;{ }^{* * *} P<0.001$ compared between indicated groups. (c) BHK cells were treated with wtSOD1 and mhSOD1/C111S for $3 \mathrm{~h}$, followed by UV radiation for $20 \mathrm{~min}$, and DNA damage was analyzed by agarose gel electrophoresis. Data are representative of three experiments.

metabolism. Although some SOD1 drugs have been developed for clinics to treat a wide spectrum of human diseases, including Peyronie's disease [29], rheumatoid arthritis [30], and acute lung injury [31], without demonstrable toxicities [32], unfortunately, large-scale manufacturing and clinical use of hSOD1 are still limited because of the challenge of rapid and inexpensive production of high-quality hSOD1 in recombinant forms. Thus, great attention has been given to the development of efficient and viable production systems to obtain hSOD1 with higher yields or activity [7, 8]. Eukaryotic expression systems are suitable systems for producing hSOD1 and dozens of groups have made progress on SOD1 preparation [18, 33-35]. For example, Cozzolino et al. successfully expressed mutant hSOD1 in NSC-34 cells and confirmed that 
removal of Cys111 remarkably improves the solubility of SOD1 [18]. However, it is difficult to be industrialized because the expression efficiency is low and thus the cost of production is high and on top of that the mammalian cell handling process is complex.

Although E. coli usually expresses recombinant protein more efficiently, the congenital deficiency in redox environment of E. coli makes it often express proteins as inclusion bodies and it is time-consuming to refold the products. As a result, increasing the soluble expression levels of recombinant proteins is a promising strategy that can increase the yields in E. coli. To study the role of the free cysteines of recombinant hSOD1 in soluble expression and yield in E. coli, we constructed and expressed three mutant hSOD1s, in which free cysteines were substituted with serines. We have verified that it is an effective stabilization strategy to replace cysteine with serine in hSOD1 so as to prevent the formation of unwanted intermolecular or intramolecular disulfide bonds and to raise the soluble protein expression levels in E. coli. Among these mutant SOD1s, the substitution of Cys111 (mhSOD1/C111S) increased significantly soluble hSOD1 expression in $E$. coli (the soluble expression of $\mathrm{mhSOD} 1 / \mathrm{C} 111 \mathrm{~S}$ is 2.35 times that of wtSOD1). The substitution of the internal Cys6 residue, however, reduced significantly the expression of soluble protein (soluble expression of mhSOD1/C6S is 0.58 times that of wtSOD1). Although the only substitution of Cys 6 reduced soluble expression remarkably, an overall increase can be observed (soluble expression of mhSOD1/C6S/C111S is 2.2 times that of wtSOD1) when Cys111 is simultaneously substituted with another amino acid. The purified protein yields of wild-type and mutant hSOD1s were also measured. These results proved that increasing soluble expression levels can increase hSOD1 yields. In addition, the mhSOD1 with higher soluble expression levels also produced stronger activity than the wtSOD1. The recombinant mhSOD1/C111S showed lower toxicity but stronger whitening and antiradiation activities than those of wtSOD1. All these data collectively demonstrate that substitution of cysteine with serine in human SOD1 is an effective strategy to develop new SOD1-associated reagents and that $\mathrm{mhSOD} 1 / \mathrm{C} 111 \mathrm{~S}$ is a promising candidate for largescale production.

\section{Competing Interests}

The authors have no conflict of interests.

\section{Acknowledgments}

This work was supported by the Science Foundation of Shaanxi Academy of Sciences (no. 2016K-05); the Science and Technology Platform Programs of Shaanxi Academy of Sciences (2015K-33); the Western Light Talent Culture Project of the Chinese Academy of Sciences (no. 2009DF03); the National Natural Science Foundation of China (no. 31200094).

\section{References}

[1] S.-G. Jeong and G.-W. Cho, "Endogenous ROS levels are increased in replicative senescence in human bone marrow mesenchymal stromal cells," Biochemical and Biophysical Research Communications, vol. 460, no. 4, pp. 971-976, 2015.
[2] E. D. Getzoff, J. A. Tainer, P. K. Weiner, P. A. Kollman, J. S. Richardson, and D. C. Richardson, "Electrostatic recognition between superoxide and copper, zinc superoxide dismutase," Nature, vol. 306, no. 5940, pp. 287-290, 1983.

[3] J. J. P. Perry, D. S. Shin, E. D. Getzoff, and J. A. Tainer, "The structural biochemistry of the superoxide dismutases," Biochimica et Biophysica Acta-Proteins and Proteomics, vol. 1804, no. 2, pp. 245-262, 2010.

[4] L. N. Maslov, N. V. Naryzhnaia, I. K. Podoksenov et al., "Reactive oxygen species are triggers and mediators of an increase in cardiac tolerance to impact of ischemia-reperfusion," Rossiǔskii Fiziologicheskǐ Zhurnal Imeni I.M. Sechenova/Rossiǔskaia Akademiia Nauk, vol. 101, no. 1, pp. 3-24, 2015.

[5] Z. Gao, E. H. Sarsour, A. L. Kalen, L. Li, M. G. Kumar, and P. C. Goswami, "Late ROS accumulation and radiosensitivity in SOD1-overexpressing human glioma cells," Free Radical Biology and Medicine, vol. 45, no. 11, pp. 1501-1509, 2008.

[6] Q. Gu, T. Feng, H. Cao et al., "HIV-TAT mediated protein transduction of $\mathrm{Cu} / \mathrm{Zn}$-superoxide dismutase-1 $\left(\mathrm{SOD}_{1}\right)$ protects skin cells from ionizing radiation," Radiation Oncology, vol. 8, no. 1, article 253, 2013.

[7] E. D. Getzoff, D. E. Cabelli, C. L. Fisher et al., "Faster superoxide dismutase mutants designed by enhancing electrostatic guidance," Nature, vol. 358, no. 6384, pp. 347-351, 1992.

[8] R. A. Hallewell, I. Laria, A. Tabrizi et al., "Genetically engineered polymers of human CuZn superoxide dismutase. Biochemistry and serum half-lives," Journal of Biological Chemistry, vol. 264, no. 9, pp. 5260-5268, 1989.

[9] X. Xue, Z. Wang, Z. Yan, J. Shi, W. Han, and Y. Zhang, "Production and purification of recombinant human BLyS mutant from inclusion bodies," Protein Expression and Purification, vol. 42, no. 1, pp. 194-199, 2005.

[10] K. Wu, X. Xue, Z. Wang et al., "Construction, purification, and immunogenicity of recombinant cystein-cystein type chemokine receptor 5 vaccine," Protein Expression and Purification, vol. 49, no. 1, pp. 108-113, 2006.

[11] Y. Wan, X. Xue, M. Li et al., "Prepared and screened a modified TNF- $\alpha$ molecule as TNF- $\alpha$ autovaccine to treat LPS induced endotoxic shock and TNF- $\alpha$ induced cachexia in mouse," Cellular Immunology, vol. 246, no. 2, pp. 55-64, 2007.

[12] M. V. Trivedi, J. S. Laurence, and T. J. Siahaan, "The role of thiols and disulfides on protein stability," Current Protein \& Peptide Science, vol. 10, no. 6, pp. 614-625, 2009.

[13] M. Trivedi, R. A. Davis, Y. Shabaik et al., "The role of covalent dimerization on the physical and chemical stability of the EC1 domain of human E-cadherin," Journal of Pharmaceutical Sciences, vol. 98, no. 10, pp. 3562-3574, 2009.

[14] I. Fridovich, "Superoxide dismutases," Advances in Enzymology and Related Areas of Molecular Biology, vol. 58, pp. 61-97, 1986.

[15] F. Arnesano, L. Banci, I. Bertini, M. Martinelli, Y. Furukawa, and T. V. O'Halloran, “The unusually stable quaternary structure of human $\mathrm{Cu}, \mathrm{Zn}$-superoxide dismutase 1 is controlled by both metal occupancy and disulfide status," Journal of Biological Chemistry, vol. 279, no. 46, pp. 47998-48003, 2004.

[16] C. L. Fisher, D. E. Cabelli, J. A. Tainer, R. A. Hallewell, and E. D. Getzoff, "The role of arginine 143 in the electrostatics and mechanism of $\mathrm{Cu}, \mathrm{Zn}$ superoxide dismutase: computational and experimental evaluation by mutational analysis," Proteins, vol. 19, no. 1, pp. 24-34, 1994.

[17] Y. Furukawa, R. Fu, H.-X. Deng, T. Siddique, and T. V. O'Halloran, "Disulfide cross-linked protein represents a significant fraction of ALS-associated $\mathrm{Cu}, \mathrm{Zn}$-superoxide dismutase 
aggregates in spinal cords of model mice," Proceedings of the National Academy of Sciences of the United States of America, vol. 103, no. 18, pp. 7148-7153, 2006.

[18] M. Cozzolino, I. Amori, M. G. Pesaresi, A. Ferri, M. Nencini, and M. T. Carrì, "Cysteine 111 affects aggregation and cytotoxicity of mutant $\mathrm{Cu}, \mathrm{Zn}$-superoxide dismutase associated with familial amyotrophic lateral sclerosis," Journal of Biological Chemistry, vol. 283, no. 2, pp. 866-874, 2008.

[19] D. W. Cleveland and J. Liu, "Oxidation versus aggregation-how do SOD1 mutants cause ALS?" Nature Medicine, vol. 6, no. 12, pp. 1320-1321, 2000.

[20] J. Hong, A. A. Moosavi-Movahedi, H. Ghourchian, M. Amani, M. Amanlou, and F. C. Chilaka, "Thermal dissociation and conformational lock of superoxide dismutase," Journal of Biochemistry and Molecular Biology, vol. 38, no. 5, pp. 533-538, 2005.

[21] I.-M. Ahl, M. J. Lindberg, and L. A. E. Tibell, "Coexpression of yeast copper chaperone (yCCS) and CuZn-superoxide dismutases in Escherichia coli yields protein with high copper contents," Protein Expression and Purification, vol. 37, no. 2, pp. 311-319, 2004.

[22] L. I. Bruijn, M. K. Houseweart, S. Kato et al., "Aggregation and motor neuron toxicity of an ALS-linked SOD1 mutant independent from wild-type SOD1," Science, vol. 281, no. 5384, pp. 1851-1854, 1998.

[23] W. Bruening, J. Roy, B. Giasson, D. A. Figlewicz, W. E. Mushynski, and H. D. Durham, "Up-regulation of protein chaperones preserves viability of cells expressing toxic $\mathrm{Cu} / \mathrm{Zn}$ superoxide dismutase mutants associated with amyotrophic lateral sclerosis," Journal of Neurochemistry, vol. 72, no. 2, pp. 693-699, 1999.

[24] J. A. Johnston, M. J. Dalton, M. E. Gurney, and R. R. Kopito, "Formation of high molecular weight complexes of mutant $\mathrm{Cu}, \mathrm{Zn}$-superoxide dismutase in a mouse model for familial amyotrophic lateral sclerosis," Proceedings of the National Academy of Sciences of the United States of America, vol. 97, no. 23, pp. 12571-12576, 2000.

[25] X.-C. Xue, Z. Yan, W.-N. Li et al., "Construction, expression, and characterization of thymosin alpha 1 tandem repeats in Escherichia coli," BioMed Research International, vol. 2013, Article ID 720285, 6 pages, 2013.

[26] S.-B. Li, H.-L. Nie, H.-T. Zhang, Y. Xue, and L.-M. Zhu, "Kinetic evaluation of aminoethylisothiourea on mushroom tyrosinase activity," Applied Biochemistry and Biotechnology, vol. 162, no. 3, pp. 641-653, 2010.

[27] L.-P. Xie, Q.-X. Chen, H. Huang, X.-D. Liu, H.-T. Chen, and R.$\mathrm{Q}$. Zhang, "Inhibitory effects of cupferron on the monophenolase and diphenolase activity of mushroom tyrosinase," International Journal of Biochemistry and Cell Biology, vol. 35, no. 12, pp. 1658-1666, 2003.

[28] J. R. Hartman, T. Geller, Z. Yavin et al., "High-level expression of enzymatically active human $\mathrm{Cu} / \mathrm{Zn}$ superoxide dismutase in Escherichia coli," Proceedings of the National Academy of Sciences of the United States of America, vol. 83, no. 19, pp. 7142-7146, 1986.

[29] H. Gustafson, B. Johansson, and F. Edsmyr, "Peyronie’s disease: experience of local treatment with Orgotein," European Urology, vol. 7, no. 6, pp. 346-348, 1981.

[30] K.-M. Goebel, U. Storck, and F. Neurath, "Intrasynovial orgotein therapy in rheumatoid arthritis," The Lancet, vol. 317, no. 8228 , pp. 1015-1017, 1981.
[31] M.-P. Lu, L.-Z. Du, W.-Z. Gu, Z.-Z. Yu, X.-X. Chen, and Z.-S. Yu, "Anti-inflammation and anti-oxidation effects of recombinant human superoxide dismutase on acute lung injury induced by meconium aspiration in infant rats," Zhejiang Da Xue Xue Bao Yi Xue Ban, vol. 34, no. 1, pp. 55-59, 2005.

[32] S. Carson, E. E. Vogin, W. Huber, and T. L. Schulte, "Safety tests of orgotein, an antiinflammatory protein," Toxicology and Applied Pharmacology, vol. 26, no. 2, pp. 184-202, 1973.

[33] E. M. Gazdag, I. C. Cirstea, R. Breitling, J. Lukeš, W. Blankenfeldt, and K. Alexandrov, "Purification and crystallization of human $\mathrm{Cu} / \mathrm{Zn}$ superoxide dismutase recombinantly produced in the protozoan Leishmania tarentolae," Acta Crystallographica Section F: Structural Biology and Crystallization Communications, vol. 66, no. 8, pp. 871-877, 2010.

[34] V. K. Bhogaraju, M. S. Levi, R. L. Reed, and J. P. Crow, "Rapid one-step purification of native dimeric ALS-associated human $\mathrm{Cu} / \mathrm{Zn}$ superoxide dismutase from transgenic rat tissues," Amyotrophic Lateral Sclerosis, vol. 11, no. 3, pp. 283-288, 2010.

[35] N. S. Park, K. S. Lee, H. D. Sohn et al., "Molecular cloning, expression, and characterization of the $\mathrm{Cu}, \mathrm{Zn}$ superoxide dismutase (SOD1) gene from the entomopathogenic fungus Cordyceps militaris," Mycologia, vol. 97, no. 1, pp. 130-138, 2005. 

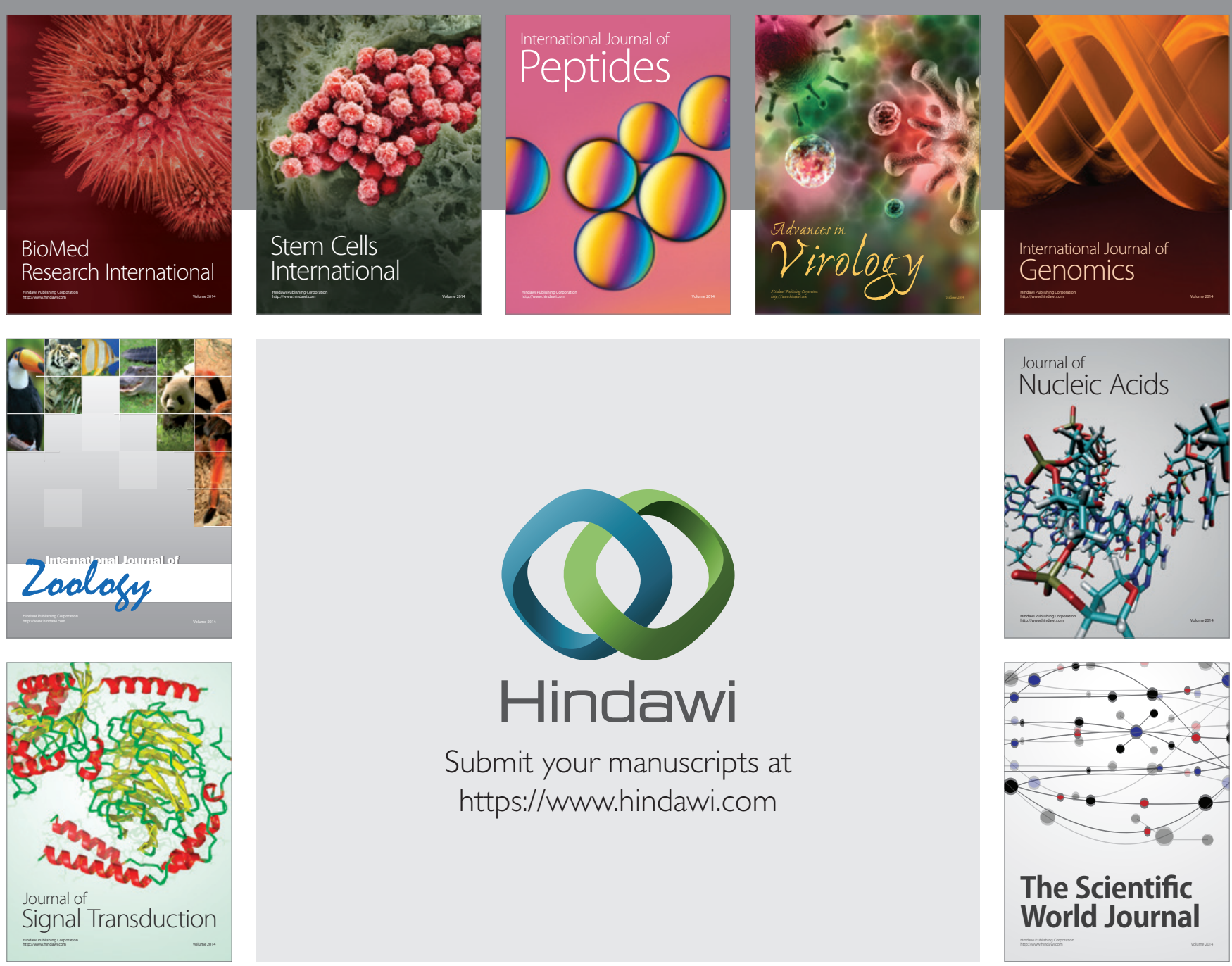

Submit your manuscripts at

https://www.hindawi.com
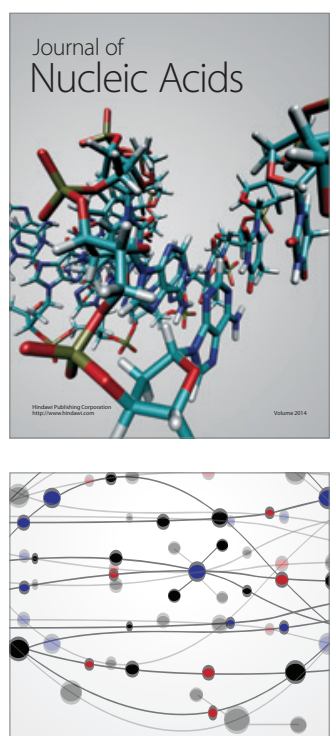

The Scientific World Journal
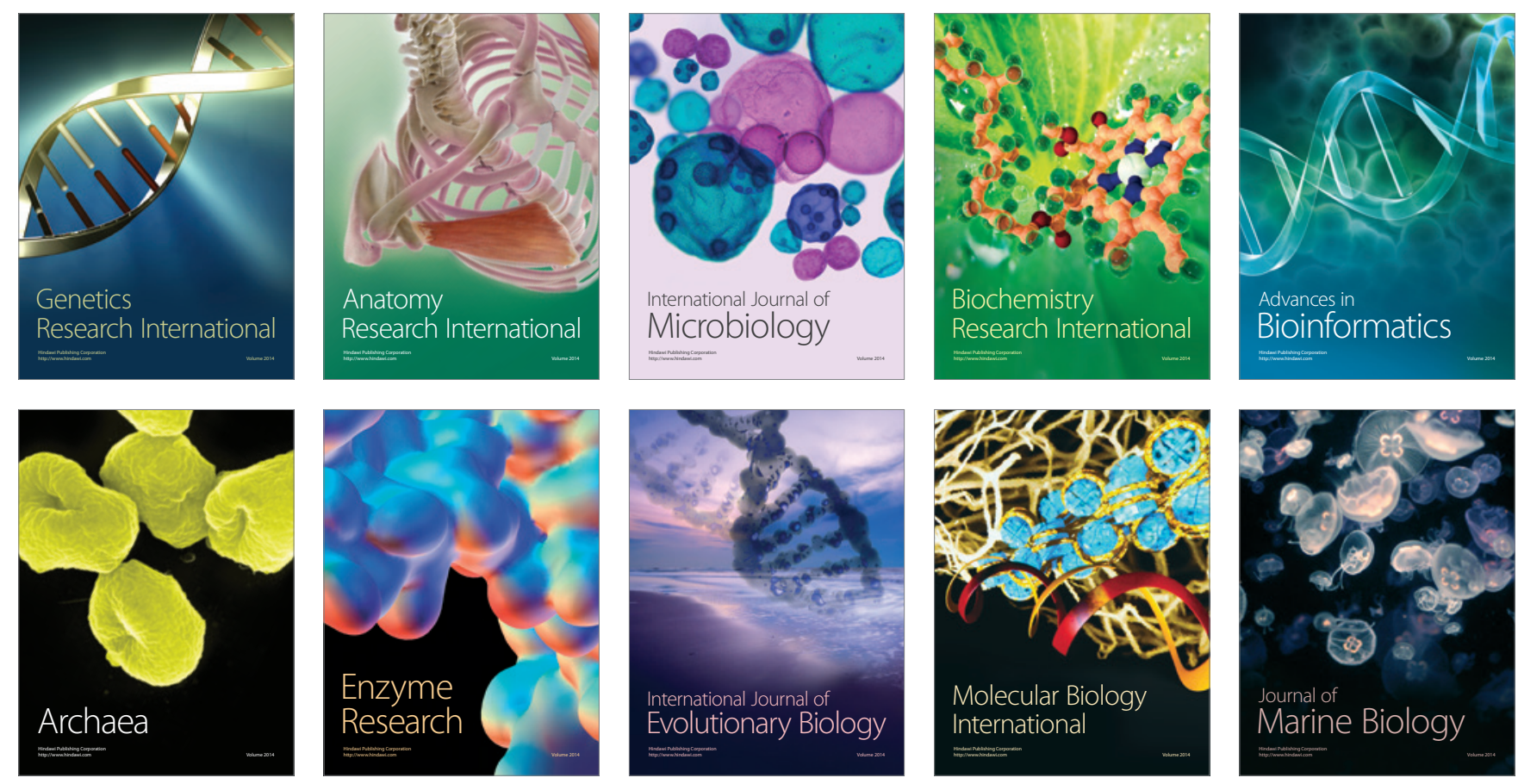